Article

\title{
Convergence and Best Proximity Points for Generalized Contraction Pairs
}

\author{
Slah Sahmim 1,*(D), Abdelbasset Felhi ${ }^{2}$ and Hassen Aydi ${ }^{3}$ (D) \\ 1 Department of Mathematics and Statistics, College of Sciences, King Faisal University, P.O. Box 400, \\ Hafouf 31982, Saudi Arabia \\ 2 Department of Mathematics and Physics Preparatory Engineering Institute, Carthage University, \\ Bizerte 7021, Tunisia; abdelbassetfelhi@gmail.com or abdelbasset.felhi@ipeib.rnu.tn \\ 3 Department of Mathematics, College of Education of Jubail, Imam Abdulrahman Bin Faisal University, \\ P.O. 12020, Industrial Jubail 31961, Saudi Arabia; hmaydi@iau.edu.sa or hassen.aydi@isima.rnu.tn \\ * Correspondence: ssahmim@kfu.edu.sa
}

Received: 12 December 2018; Accepted: 8 February 2019; Published: 15 February 2019

\begin{abstract}
This paper is devoted to studying the existence of best proximity points and convergence for a class of generalized contraction pairs by using the concept of proximally-complete pairs and proximally-complete semi-sharp proximinal pairs. The obtained results are generalizations of the result of Sadiq Basha (Basha, S., Best proximity points: global optimal approximate solutions, J. Glob. Optim. 2011, 49, 15-21) As an application, we give a result for nonexpansive mappings in normed vector spaces.
\end{abstract}

Keywords: contraction pair; proximally-complete pair; semi-sharp proximinal; best proximity point; nonexpansive mapping

2000 MSC: 47H09; 47H10; 54H25

\section{Introduction and Preliminaries}

Let $(X, d)$ be a metric space. Consider two nonempty subsets $P$ and $Q$ of $X$. Given a non-self mapping $f: P \rightarrow Q$, then if $P \cap f(P)=\varnothing$, the fixed point equation $f(t)=t$ has no solution, that is $d(t, f(t))>0$ for all $t$ in $P$. The object of best proximity theory is to locate $z \in P$ such that $d(z, f(z))$ is minimum and so as to ensure the existence of a point $a \in X$ verifying $d(a, f(a))=d(P, Q)$, where $d(P, Q)=\inf \{d(\xi, \vartheta): \xi \in P, \vartheta \in Q\}$. In this case, $a$ is called a best proximal point of $f$. Best proximity point theorems furnish sufficient conditions yielding the existence of approximate solutions, which are optimal, as well. The investigation of best proximity points is an attractive topic for optimization theory; see [1-33]. Consider:

$$
P_{0}=\{\xi \in P: d(\xi, \vartheta)=d(P, Q), \text { for some } \vartheta \in Q\}
$$

and:

$$
Q_{0}=\{\vartheta \in Q: d(\xi, \vartheta)=d(P, Q) \text {, for some } \xi \in P\} .
$$

In the case that $P \cap Q \neq \varnothing$, the subsets $P_{0}$ and $Q_{0}$ are nonempty. Moreover, if $P_{0}$ or $Q_{0}$ is nonempty, then again, $P_{0}$ and $Q_{0}$ are nonempty. In the same direction, the following lemma gives some sufficient conditions in the case of reflexive Banach spaces.

Lemma 1 ([18]). Let $P$ be a nonempty, bounded, closed, and convex subset of a reflexive Banach space X. Then, $P_{0}$ and $Q_{0}$ are nonempty. 
Let $\mathbb{N}^{*}($ resp. $\mathbb{N}$ ) be the set of positive (resp. nonnegative) integers. In [3], Sadiq Basha proved the following result.

Theorem 1 ([3]). Let $P$ and $Q$ be two nonempty compact subsets of a metric space $(X, d)$. Suppose that $f: P \rightarrow Q$ and $g: Q \rightarrow P$ are two mappings satisfying the following conditions:

(i) $f$ and $g$ are contractive;

(ii) $d(f \xi, g \vartheta)<d(\xi, \vartheta)$ whenever $d(P, Q)<d(\xi, \vartheta)$ for $(\xi, \vartheta) \in P \times Q$.

Then, there exist $z \in P$ and $w \in Q$ such that:

$$
d(z, f z)=d(w, g w)=d(z, w)=d(P, Q) .
$$

Further, for an arbitrary element $\xi_{0} \in P_{0}$, let $\xi_{2 n+1}=f \xi_{2 n}$ and $\xi_{2 n}=g \xi_{2 n-1}$ for $n \geq 1$. Then, $\left(\xi_{2 n}\right)$ converges to $z$, and $\left(\xi_{2 n+1}\right)$ converges to $w$.

The concept of proximally complete pairs was first initiated by Espínola et al. [9] and was used to study the existence and convergence to best proximity points for cyclic contraction mappings.

Definition 1 ([14]). Let $P$ and $Q$ be nonempty subsets of a metric space $(X, d)$. Let $\left(\xi_{n}\right)$ be a sequence in $P \cup Q$ such that $\left(\xi_{2 n}\right)$ in $P$ and $\left(\xi_{2 n+1}\right)$ in $Q$ for $n \geq 0$. If for each $\epsilon>0$, there exists an integer $n_{0}$ such that for all even integers $p \geq n_{0}$ and odd integers $q \geq n_{0}, d\left(\xi_{p}, \xi_{q}\right)<d(P, Q)+\epsilon$, then $\left(\xi_{n}\right)$ is called a cyclical Cauchy sequence.

Lemma 2 ([9]). (i) The sequence $\left(\xi_{n}\right)$ in $P \cup Q$ such that $\left(\xi_{2 n}\right)$ in $P$ and $\left(\xi_{2 n+1}\right)$ in $Q$ for $n \geq 0$ is cyclical Cauchy if:

$$
\lim _{n, m \rightarrow \infty} d\left(\xi_{2 n}, \xi_{2 m+1}\right)=d(P, Q) .
$$

(ii) Any cyclical Cauchy sequence can have more than one accumulation point.

Example 1. We endow on $X=\mathbb{R}^{2}$ the metric:

$$
d\left(\left(\xi_{1}, \vartheta_{1}\right),\left(\xi_{2}, \vartheta_{2}\right)\right)=\left|\xi_{1}-\xi_{2}\right|+\left|\vartheta_{1}-\vartheta_{2}\right| .
$$

Let $P=\{(1, u):-2 \leq u \leq 2\}$ and $Q=\{(0, u):-1 \leq u \leq 1\}$. Consider the sequence $\left(\theta_{n}\right)_{n \geq 0}$ defined by $\theta_{n}=\left(\frac{1+(-1)^{n}}{2}, 1+\frac{(-1)^{n}}{n+1}\right)$. Then, $\theta_{2 n}=\left(1,1+\frac{1}{2 n+1}\right)$ and $\theta_{2 n+1}=\left(0,1-\frac{1}{2 n+1}\right)$, so $\left(\theta_{2 n}\right)$ is in $P$ and $\left(\theta_{2 n+1}\right)$ is in $Q$. Furthermore, $\lim _{n \rightarrow \infty} \theta_{2 n}=(1,1) \in P$ and $\lim _{n \rightarrow \infty} \theta_{2 n+1}=(0,1) \in Q$. Then, $\left(\theta_{n}\right)$ does not converge. Moreover,

$$
\lim _{n, m \rightarrow \infty} d\left(\theta_{2 n}, \theta_{2 m+1}\right)=\lim _{n, m \rightarrow \infty}\left(1+\left|\frac{1}{2 n+1}+\frac{1}{2 m+2}\right|\right)=1=d(P, Q) .
$$

Thus, the sequence $\left(\theta_{n}\right)$ is cyclical Cauchy.

Lemma 3 ([9]). Let $(X, d)$ be a metric space. Given $P$ and $Q$ two nonempty subsets of $X$, then:

(i) Every cyclical Cauchy sequence is bounded.

(ii) If $d(P, Q)=0$, then every cyclical Cauchy sequence $\left(\xi_{n}\right) \subseteq P \cup Q$ is a Cauchy sequence.

Definition 2 ([9]). Let $P$ and $Q$ be nonempty subsets of a metric space $(X, d)$. The pair $(P, Q)$ is called proximally complete if, for every cyclically Cauchy sequence $\left(\xi_{n}\right) \subseteq P \cup Q,\left(\xi_{2 n}\right)$ and $\left(\xi_{2 n+1}\right)$ have convergent subsequences in $P$ and $Q$, respectively.

In the following, we give cases where the pair $(P, Q)$ is proximally complete. 
Theorem 2 ([9]). Let $(X, d)$ be a metric space. Let $P$ and $Q$ be nonempty subsets of $X$. We have:

(i) If $(P, Q)$ is a boundedly-compact pair, then it is proximally complete.

(ii) If $(P, Q)$ is a closed pair such that $d(P, Q)=0$ and $(X, d)$ is complete, then $(P, Q)$ is proximally complete.

Theorem 3 ([9]). Consider a uniformly-convex Banach space $(X, \| .||)$. Then, any nonempty, closed and convex pair $(P, Q)$ of $X$ is proximally complete.

Theorem 4 ([9]). If $(P, Q)$ is a proximally-complete pair of a metric space $X$, then the subsets $P_{0}$ and $Q_{0}$ are closed in $X$.

Definition 3 ([9]). Let $P$ and $Q$ be nonempty subsets of a metric space $(X, d)$. The pair $(P, Q)$ is called semi-sharp proximinal if, for all $\xi \in P$ and $\vartheta \in Q$, there exist at most $\xi^{\star} \in Q$ and at most $\vartheta^{\star} \in P$ such that $d\left(\xi, \xi^{\star}\right)=d\left(\vartheta^{\star}, \vartheta\right)=d(P, Q)$.

Example 2 ([19]). Let $(X,\|\|$.$) be a strictly Banach convex space. Then, every closed and convex pair (P, Q)$ of $X$ is semi-sharp proximinal.

Example 3. Consider $X=\mathbb{R}^{2}$ endowed with the metric defined by:

$$
d\left(\left(\xi_{1}, \vartheta_{1}\right),\left(\xi_{2}, \vartheta_{2}\right)\right)=\left|\xi_{1}-\xi_{2}\right|+\left|\vartheta_{1}-\vartheta_{2}\right| .
$$

Let $P=\{(1,2),(2,2)\}$ and $Q=\{(2,1),(1,1)\}$. We have $d(P, Q)=1$. Furthermore,

$$
d((1,2),(1,1))=d((2,2),(2,1))=1, d((1,2),(2,1))=d((2,2),(1,1))=2 .
$$

Then, $(P, Q)$ is semi-sharp proximinal.

Definition 4 ([34]). A nonnegative function $\varphi$ defined on $[0, \infty)$ is said to be a $(c)$-comparison function if: $\left(\varphi_{1}\right) \varphi$ is non-decreasing;

$\left(\varphi_{2}\right)$ there are $p_{0} \in \mathbb{N}$ and $r \in(0,1)$ so that for $p \geq p_{0}$ and $s>0$,

$$
\varphi^{p+1}(s) \leq r \varphi^{p}(s)+u_{p}
$$

where the series $\sum_{p=1}^{\infty} u_{p}$ is convergent and $u_{p} \geq 0 . \varphi^{p}$ is the $p^{\text {th }}$ iterate of $\varphi$.

Lemma 4 ([34]). Let $\varphi:[0, \infty) \rightarrow[0, \infty)$ be a (c)-comparison function. Then,

(i) $\left(\varphi^{n}(s)\right)_{n \in \mathbb{N}}$ converges to zero as $n \rightarrow \infty$, for each $s>0$;

(ii) $\varphi(s)<$ for each $s>0$;

(iii) $\varphi$ is continuous at zero, and $\varphi(0)=0$;

(iv) the series $\sum_{n=0}^{\infty} \varphi^{n}(s)<\infty$ for each $t>0$.

In the paper of Sadiq Bacha [3], the two considered mappings are supposed to be contractive. While in this paper (Theorem 5), the contractivity of mappings and Condition (b) in Theorem 2.1 of [3] are omitted. We just take weaker hypotheses, and we get the same result by considering proximally-complete pairs or proximally-complete semi-sharp proximinal pairs. We give conditions ensuring the existence of best proximity points via contraction pairs. We also provide a result for nonexpansive mappings in normed vector spaces. The obtained results are supported by some examples.

\section{Main Results}

The first theorem is: 
Theorem 5. Let $(P, Q)$ be a proximally-complete pair in a metric space $(X, d)$. Let $f: P \rightarrow Q$ and $g: Q \rightarrow P$ be non-self mappings such that for all $(x, y) \in P \times Q$,

$$
D(f x, g y) \leq \varphi(D(x, y)),
$$

where $\varphi$ is a c-comparison function and $D(x, y):=d(x, y)-d(P, Q)$.

Then, there exist $\xi_{\star} \in P$ and $\vartheta_{\star} \in Q$ such that:

$$
d\left(\xi_{\star}, f \xi_{\star}\right)=d\left(\vartheta_{\star}, g \vartheta_{\star}\right)=d\left(\xi_{\star}, \vartheta_{\star}\right)=d(P, Q) .
$$

Proof. Let $\xi_{0} \in P$. Define the sequence $\left(\xi_{n}\right)$ in $P \cup Q$ as follows:

$$
\xi_{2 n+1}=f \xi_{2 n} \quad \text { and } \quad \xi_{2 n}=g \xi_{2 n-1}, \quad n=1,2, \cdots
$$

By (4), we have:

$$
D\left(\xi_{1}, \xi_{2}\right)=D\left(f \xi_{0}, g \xi_{1}\right) \leq \varphi\left(D\left(\xi_{0}, \xi_{1}\right)\right)
$$

Again:

$$
D\left(\xi_{2}, \xi_{3}\right)=D\left(f \xi_{2}, g \xi_{1}\right) \leq \varphi\left(D\left(\xi_{2}, \xi_{1}\right)\right) \leq \varphi^{2}\left(D\left(\xi_{0}, \xi_{1}\right)\right) .
$$

Continuing in this way, we find that:

$$
D\left(\xi_{n}, \xi_{n+1}\right) \leq \varphi^{n}\left(D\left(\xi_{0}, \xi_{1}\right)\right) \quad \forall n \geq 0 .
$$

If $d(P, Q)=0$, it is easy to show that there exists $x \in P \cap Q$ such that $d(x, f x)=d(x, g x)=0=$ $d(P, Q)$. Moreover, the sequence $\left(\xi_{n}\right)$ converges to a common fixed point of $f$ and $g$.

From now on, suppose that $d(P, Q)>0$. If $D\left(\xi_{2 n}, \xi_{2 n+1}\right)=0$ for some $n$, then $\xi_{2 n}$ is a best proximity point of $f$. From (4), we have:

$$
D\left(\xi_{2 n+1}, g \xi_{2 n+1}\right)=D\left(f \xi_{2 n}, g \xi_{2 n+1}\right) \leq \varphi\left(D\left(\xi_{2 n}, \xi_{2 n+1}\right)\right)=\varphi(0)=0
$$

and so, $\xi_{2 n+1}$ is a best proximity point of $g$. Similarly, if $D\left(\xi_{2 n+1}, \xi_{2 n+2}\right)=0$ for some $n$, then $\xi_{2 n+1}$ is a best proximity point of $g$, and $\xi_{2 n+2}$ is a best proximity point of $f$.

Suppose now that $D\left(\xi_{n}, \xi_{n+1}\right)>0$ for all $n \geq 0$. Passing to the limit in Inequality (5), we get $\lim _{n \rightarrow \infty} D\left(\xi_{n}, \xi_{n+1}\right)=0$. Hence,

$$
\lim _{n \rightarrow \infty} d\left(\xi_{n}, \xi_{n+1}\right)=d(P, Q) .
$$

We claim that $\left(\xi_{n}\right)$ is bounded. In view of (6), it suffices to prove that $\left(\xi_{2 n+1}\right)$ is bounded. We argue by contradiction. Then, there exists $N \in \mathbb{N}^{*}$ such that:

$$
d\left(\xi_{2}, \xi_{2 N+1}\right)>M \quad \text { and } d\left(\xi_{2}, \xi_{2 N-1}\right) \leq M,
$$

where the real $M>0$ is chosen in order that:

$$
M-d(P, Q)>\varphi^{2}\left(d\left(\xi_{0}, \xi_{1}\right)+\varphi\left[d\left(\xi_{0}, \xi_{1}\right)-d(P, Q)\right]+M\right) .
$$

Using (4), we have:

$$
\begin{aligned}
d\left(\xi_{2}, \xi_{2 N+1}\right)-d(P, Q) & =d\left(f \xi_{2 N}, g \xi_{1}\right)-d(P, Q) \\
& \leq \varphi\left(d\left(\xi_{2 N}, \xi_{1}\right)-d(P, Q)\right) \\
& =\varphi\left(d\left(f \xi_{0}, g \xi_{2 N-1}\right)-d(P, Q)\right) \\
& \leq \varphi^{2}\left(d\left(\xi_{0}, \xi_{2 N-1}\right)-d(P, Q)\right) .
\end{aligned}
$$


From (7), we get:

$$
\begin{aligned}
M-d(P, Q) & <\varphi^{2}\left(d\left(\xi_{0}, \xi_{2 N-1}\right)-d(P, Q)\right) \\
& \leq \varphi^{2}\left(d\left(\xi_{0}, \xi_{1}\right)+d\left(\xi_{1}, \xi_{2}\right)+d\left(\xi_{2}, \xi_{2 N-1}\right)-d(P, Q)\right) \\
& =\varphi^{2}\left(d\left(\xi_{0}, \xi_{1}\right)+d\left(f \xi_{0}, g \xi_{1}\right)+d\left(\xi_{2}, \xi_{2 N-1}\right)-d(P, Q)\right) \\
& \leq \varphi^{2}\left(d\left(\xi_{0}, \xi_{1}\right)+\varphi\left[d\left(\xi_{0}, \xi_{1}\right)-d(P, Q)\right]+d\left(\xi_{2}, \xi_{2 N-1}\right)\right) \\
& \leq \varphi^{2}\left(d\left(\xi_{0}, \xi_{1}\right)+\varphi\left[d\left(\xi_{0}, \xi_{1}\right)-d(P, Q)\right]+M\right) .
\end{aligned}
$$

We deduce that:

$$
M-d(P, Q)<\varphi^{2}\left(d\left(\xi_{0}, \xi_{1}\right)+\varphi\left[d\left(\xi_{0}, \xi_{1}\right)-d(P, Q)\right]+M\right)
$$

which is a contradiction with respect to (8). Hence, $\left(\xi_{n}\right)$ is bounded.

We claim that $\left(\xi_{n}\right)$ is a cyclical Cauchy sequence. Letting $m \geq n$, we have by (4),

$$
\begin{aligned}
D\left(\xi_{2 n}, \xi_{2 m+1}\right)=D\left(f \xi_{2 m}, g \xi_{2 n-1}\right) & \leq \varphi\left(D\left(\xi_{2 m}, \xi_{2 n-1}\right)\right) \\
& \leq \varphi^{2}\left(D\left(\xi_{2 m-1}, \xi_{2 n-2}\right)\right) \\
& \vdots \\
& \leq \varphi^{2 n}\left(D\left(\xi_{0}, \xi_{2(m-n)+1}\right)\right) .
\end{aligned}
$$

Since $\left(\xi_{n}\right)$ is bounded and $\varphi$ is non-decreasing, by passing to the limit in the above inequality, we get $\lim _{m, n \rightarrow \infty} D\left(\xi_{2 n}, \xi_{2 m+1}\right)=0$, which implies that:

$$
\lim _{n, m \rightarrow \infty} d\left(\xi_{2 n}, \xi_{2 m+1}\right)=d(P, Q)
$$

Then, $\left(\xi_{n}\right)$ is a cyclical Cauchy sequence. Since $(P, Q)$ is a proximally-complete pair, the sequence $\left(\xi_{n}\right)$ has a subsequence $\left(\xi_{2 n_{k}}\right)$ converging to some element $\xi_{\star} \in P$. Again, $\left(\xi_{n}\right)$ has a convergent subsequence $\left(\xi_{2 m_{k}+1}\right)$ to some $\vartheta_{\star} \in Q$.

We claim that $\xi_{\star}$ is a best proximity of $f$. We have:

$$
D\left(\xi_{\star}, \xi_{2 n_{k}-1}\right)=d\left(\xi_{\star}, \xi_{2 n_{k}-1}\right)-d(P, Q) \leq d\left(\xi_{\star}, \xi_{2 n_{k}}\right)+d\left(\xi_{2 n_{k}}, \xi_{2 n_{k}-1}\right)-d(P, Q) .
$$

Using (6), we obtain:

$$
\lim _{k \rightarrow \infty} D\left(\xi_{\star}, \xi_{2 n_{k}-1}\right)=0
$$

By (4),

$$
D\left(\xi_{2 n_{k}}, f \xi_{\star}\right)=D\left(f \xi_{\star}, g \xi_{2 n_{k}-1}\right) \leq \varphi\left(D\left(\xi_{\star}, \xi_{2 n_{k}-1}\right)\right) .
$$

Taking $k \rightarrow \infty$ and using (10) together with the fact that $\varphi$ is continuous at zero, we obtain that:

$$
D\left(\xi_{\star}, f \xi_{\star}\right)=\lim _{k \rightarrow \infty} D\left(\xi_{2 n_{k}}, f \xi_{\star}\right)=\varphi(0)=0,
$$

which implies that $D\left(\xi_{\star}, f \xi_{\star}\right)=0$, and so, $d\left(\xi_{\star}, f \xi_{\star}\right)=d(P, Q)$. Similarly, $\vartheta_{\star}$ is a best proximity of $g$, i.e., $d\left(\vartheta_{\star}, g \vartheta_{\star}\right)=d(P, Q)$. From $(9)$, we have $d\left(\xi_{\star}, \vartheta_{\star}\right)=d(P, Q)$.

The following illustrates Theorem 5. 
Example 4. Consider $X=\mathbb{R}^{2}$ with the metric defined as $d\left(\left(\xi_{1}, \vartheta_{1}\right),\left(\xi_{2}, \vartheta_{2}\right)\right)=\left|\xi_{1}-\xi_{2}\right|+\left|\vartheta_{1}-\vartheta_{2}\right|$. Let $P=\{1\} \times[0,1]$ and $Q=\{0\} \times[0,1]$. Note that $d(P, Q)=1$ and $(P, Q)$ is a proximally-complete pair. For $x \in[0,1]$, define $f: P \rightarrow Q$ and $g: Q \rightarrow P$ as follows:

$$
f(1, x)=\left(0, \frac{x^{2}+1}{4}\right) \text { and } g(0, x)=\left(1, \frac{x^{2}+1}{4}\right) .
$$

Taking $x, y \in[0,1]$, one writes:

$$
\begin{aligned}
D(f(1, x), g(0, y))=d(f(1, x), g(0, y))-1 & =\frac{1}{4}\left|x^{2}-y^{2}\right|=\frac{1}{4}(x+y)|x-y| \\
& \leq \frac{1}{2}|x-y|=\frac{1}{2}(d((1, x),(0, y))-1) \\
& =\frac{1}{2} D((1, x),(0, y)) .
\end{aligned}
$$

Then, the condition contraction (4) is verified with $\varphi(t)=\frac{1}{2} t$. Hence, $f$ has a best proximity in $P$, and $g$ has a best proximity in $Q$. Here, $(1,2-\sqrt{3})$ is the unique best proximity of $f$ and $(0,2-\sqrt{3})$ is the unique best proximity of $g$. Furthermore, $d((1,2-\sqrt{3}),(0,2-\sqrt{3}))=1=d(P, Q)$.

The following results are simple consequences of Theorem 5 . We omit their proofs.

Corollary 1. Let $(P, Q)$ be a proximally-complete pair in a metric space $(X, d)$. Let $f: P \rightarrow Q$ and $g: Q \rightarrow P$ be non-self maps such that for all $(x, y) \in P \times Q$,

$$
d(f x, g y) \leq \lambda d(x, y)+(1-\lambda) d(P, Q),
$$

where $\lambda \in[0,1)$. Then, there are $\xi_{\star} \in P$ and $\vartheta_{\star} \in Q$ so that:

$$
d\left(\xi_{\star}, f \xi_{\star}\right)=d\left(\vartheta_{\star}, g \vartheta_{\star}\right)=d\left(\xi_{\star}, \vartheta_{\star}\right)=d(P, Q) .
$$

Corollary 2. Let $(P, Q)$ be a proximally-complete pair in a metric space $(X, d)$. Let $f: P \cup Q \rightarrow P \cup Q$ be a non-self mapping such that $f(P) \subseteq Q, f(Q) \subseteq P$ and for all $(x, y) \in P \times Q$,

$$
D(f x, f y) \leq \varphi(D(x, y)),
$$

where $\varphi$ is a c-comparison function and $D(x, y):=d(x, y)-d(P, Q)$. Then, there are $\xi_{\star} \in P$ and $\vartheta_{\star} \in Q$, so that:

$$
d\left(\xi_{\star}, f \xi_{\star}\right)=d\left(\vartheta_{\star}, f \vartheta_{\star}\right)=d\left(\xi_{\star}, \vartheta_{\star}\right)=d(P, Q) .
$$

Corollary 3. Let $(P, Q)$ be a proximally-complete pair in a metric space $(X, d)$. Let $f: P \cup Q \rightarrow P \cup Q$ be a given non-self map such that $f(P) \subseteq Q, f(Q) \subseteq P$ and for all $(x, y) \in P \times Q$,

$$
d(f x, f y) \leq \lambda d(x, y)+(1-\lambda) d(P, Q),
$$

where $\lambda \in[0,1)$. Then, there are $\xi_{\star} \in P$ and $\vartheta_{\star} \in Q$ so that:

$$
d\left(\xi_{\star}, f \xi_{\star}\right)=d\left(\vartheta_{\star}, f \vartheta_{\star}\right)=d\left(\xi_{\star}, \vartheta_{\star}\right)=d(P, Q)
$$

Our second main result is: 
Theorem 6. Let $(P, Q)$ be a proximally-complete semi-sharp proximinal pair in a metric space $(X, d)$. Let $f$ : $P \rightarrow Q$ and $g: Q \rightarrow P$ be non-self maps such that for all $(x, y) \in P \times Q$,

$$
D(f x, g y) \leq \varphi(D(x, y)),
$$

where $\varphi$ is a c-comparison function and $D(x, y):=d(x, y)-d(P, Q)$. Then, the following hold:

(i) There is $\xi_{\star} \in P$ such that $d\left(\xi_{\star}, f \xi_{\star}\right)=d(P, Q)$;

(ii) $\xi_{\star}$ is a fixed point of $g f$, i.e., $g f \xi_{\star}=\xi_{\star}$, and $f \xi_{\star}$ is a fixed point of $f g$, i.e., $f g\left(f \xi_{\star}\right)=f \xi_{\star}$;

(iii) For any $\xi_{0} \in P$, let $\xi_{2 n+1}=f \xi_{2 n}$ and $\xi_{2 n}=g \xi_{2 n-1}$. Then, the sequence $\left(\xi_{2 n}\right)$ converges to $\xi_{\star}$, and the sequence $\left(\xi_{2 n+1}\right)$ converges to $f \xi_{\star}$.

Proof. Let $\xi_{0} \in P$. Define the sequence $\left(\xi_{n}\right)$ by $\xi_{2 n+1}=f \xi_{2 n}$ and $\xi_{2 n}=g \xi_{2 n-1}$. By Theorem 5, there exists $\left(\xi_{\star}, \vartheta_{\star}\right) \in P \times Q$ so that:

$$
d\left(\xi_{\star}, f \xi_{\star}\right)=d\left(\vartheta_{\star}, g \vartheta_{\star}\right)=d\left(\xi_{\star}, \vartheta_{\star}\right)=d(P, Q) .
$$

From (11),

$$
D\left(f \xi_{\star}, g f \xi_{\star}\right) \leq \varphi\left(D\left(\xi_{\star}, f \xi_{\star}\right)\right)=\varphi(0)=0 .
$$

Then, $D\left(f \xi_{\star}, g f \xi_{\star}\right)=0$, and so, $d\left(f \xi_{\star}, g f \xi_{\star}\right)=d\left(f \xi_{\star}, \xi_{\star}\right)=d\left(\xi_{\star}, \vartheta_{\star}\right)=d(P, Q)$. Since $(P, Q)$ is semi-sharp proximinal, then $\vartheta_{\star}=f \xi_{\star}$ and $g f \xi_{\star}=\xi_{\star}$. It follows that $f g\left(f \xi_{\star}\right)=f\left(g f \xi_{\star}\right)=f \xi_{\star}$. By Theorem 5, the sequence $\left(\xi_{n}\right)$ is cyclical Cauchy in $P \cup Q$. Furthermore, the sequence $\left(\xi_{2 n}\right)$ has a convergent subsequence $\left(\xi_{2 n_{k}}\right)$ to $\xi_{\star}$, and the sequence $\left(\xi_{2 n+1}\right)$ has a convergent subsequence $\left(\xi_{2 n_{k}+1}\right)$ to $\vartheta_{\star}=f \xi_{\star}$. Following Theorem 3.3 of [9] and since $(P, Q)$ is a semi-sharp proximinal pair, the sequence $\left(\xi_{2 n}\right)$ is Cauchy. Furthermore, $\left(\xi_{2 n}\right)$ has a convergent subsequence to $\xi_{\star}$. Then, $\left(\xi_{2 n}\right)$ converges to $\xi_{\star}$. Similarly, we show that $\left(\xi_{2 n+1}\right)$ converges to $f \xi_{\star}$.

The following examples support Theorem 6.

Example 5. Consider $X=\mathbb{R}^{2}$ with the metric defined as $d\left(\left(\xi_{1}, \vartheta_{1}\right),\left(\xi_{2}, \vartheta_{2}\right)\right)=\left|\xi_{1}-\xi_{2}\right|+\left|\vartheta_{1}-\vartheta_{2}\right|$. Let $P=\{(1,1),(1,2)\}$ and $Q=\{(2,1),(2,2)\}$. We have $d(P, Q)=1$, and $(P, Q)$ is a proximally-complete semi-sharp pair. Define $f: P \rightarrow Q$ and $g: Q \rightarrow P$ as follows:

$$
f(1,1)=f(1,2)=(2,2) \quad \text { and } \quad g(2,2)=g(2,1)=(1,2) .
$$

The condition (11) is verified for each c-comparison function $\varphi$. Here, $(1,2)$ is the unique best proximity of $f$. Furthermore, $(1,2)$ is the unique fixed point of $g f$, and $f(1,2)=(2,2)$ is the unique fixed point of $f g$. Again, if $\theta_{0}=(1,1)$ with $\theta_{2 n+1}=f \theta_{2 n}$ and $\theta_{2 n}=g \theta_{2 n-1}$, then $\theta_{2 n}=(1,2)$ for all $n \geq 1$ and $\theta_{2 n+1}=(2,2)$ for all $n \geq 0$.

Example 6. Consider the metric space $(X, d)$ given by Example 5. Consider the subsets $P=\{(s, 0), s \in[0,1]\}$ and $Q=\{(t, 1), t \in[0,1]\}$. Here, $d(P, Q)=1$. For all $x=(s, 0) \in P$ and $y=(t, 1) \in Q$, there exist $a$ unique $u=(s, 1) \in Q$ and a unique $v=(t, 0) \in P$ such that $d(x, u)=d(y, v)=1=d(P, Q)$, so $(P, Q)$ is a proximally-complete semi-sharp pair. For $s, t \in[0,1]$, define $f: P \rightarrow Q$ by $f(s, 0)=\left(\frac{s+1}{2}, 1\right)$ and $g: Q \rightarrow P$ $g(t, 1)=\left(\frac{t+1}{2}, 0\right)$. Let $x=(s, 0) \in P$ and $y=(t, 1) \in Q$, then:

$$
\begin{aligned}
D(f x, g y) & =D\left(\left(\left(\frac{s+1}{2}, 1\right),\left(\frac{t+1}{2}, 0\right)\right)\right. \\
& =\left|\frac{s-t}{2}\right|+1-d(P, Q) \\
& =\left|\frac{s-t}{2}\right| \\
& =\varphi(D(x, y) \mid
\end{aligned}
$$


where $\varphi(t)=\frac{t}{2}$. There exists a unique point $x^{\star}=(1,0) \in P$ such that $d\left(x^{\star}, f x^{\star}\right)=1=d(P, Q)$ and $g f x^{\star}=g(1,1)=x^{\star}$. Here, $(1,2)$ is the unique fixed point of $g f$ and $f(1,2)=(2,2)$ is the unique fixed point of $f g$. For any $\theta_{0}=(s, 0) \in P$, let $\theta_{2 n+1}=f \theta_{2 n}$ and $\theta_{2 n}=g \theta_{2 n-1}$. Then, the sequence $\left(\theta_{2 n}\right)$ converges to $x^{\star}$, and the sequence $\left(\theta_{2 n+1}\right)$ converges to $f x^{\star}$.

The following corollaries are consequences of Theorem 6.

Corollary 4. Let $(P, Q)$ be a proximally-complete semi-sharp proximinal pair in a metric space $(X, d)$. Let $f$ : $P \rightarrow Q$ and $g: Q \rightarrow P$ be non-self mappings such that for all $(x, y) \in P \times Q$,

$$
d(f x, g y) \leq \lambda d(x, y)+(1-\lambda) d(P, Q),
$$

where $\lambda \in[0,1)$. Then, the following hold:

(i) There exists a point $\xi_{\star} \in P$ such that $d\left(\xi_{\star}, f \xi_{\star}\right)=d(P, Q)$;

(ii) $\xi_{\star}$ is a fixed point of $g f$, i.e., $g f \xi_{\star}=\xi_{\star}$, and $f \xi_{\star}$ is a fixed point of $f g$, i.e., $f g\left(f \xi_{\star}\right)=f \xi_{\star}$;

(iii) For any $\xi_{0} \in P$, let $\xi_{2 n+1}=f \xi_{2 n}$ and $\xi_{2 n}=g \xi_{2 n-1}$. Then, the sequence $\left(\xi_{2 n}\right)$ converges to $\xi_{\star}$, and the sequence $\left(\xi_{2 n+1}\right)$ converges to $f \xi_{\star}$.

Corollary 5. Let $(P, Q)$ be a proximally-complete semi-sharp proximinal pair in a metric space $(X, d)$. Let $f$ : $P \cup Q \rightarrow P \cup Q$ be a non-self mapping such that $f(P) \subseteq Q, f(Q) \subseteq P$ and for all $(x, y) \in P \times Q$,

$$
D(f x, f y) \leq \varphi(D(x, y)),
$$

where $\varphi$ is a c-comparison function and $D(x, y):=d(x, y)-d(P, Q)$. Then, the following hold:

(i) There exists a point $\xi_{\star} \in P$ such that $d\left(\xi_{\star}, f \xi_{\star}\right)=d(P, Q)$;

(ii) $\xi_{\star}$ is a fixed point of $f^{2}$ in $P$, and $f \xi_{\star}$ is a fixed point of $f^{2}$ in $Q$;

(iii) For any $\xi_{0} \in P$, let $\xi_{n+1}=f \xi_{n}$. Then, the sequence $\left(\xi_{2 n}\right)$ converges to $\xi_{\star}$, and the sequence $\left(\xi_{2 n+1}\right)$ converges to $f \xi_{\star}$.

Corollary 6. Let $(P, Q)$ be a proximally-complete semi-sharp proximinal pair in a metric space $(X, d)$. Let $f$ : $P \cup Q \rightarrow P \cup Q$ be a non-self mapping such that $f(P) \subseteq Q, f(Q) \subseteq P$ and for all $(x, y) \in P \times Q$,

$$
d(f x, f y) \leq \lambda d(x, y)+(1-\lambda) d(P, Q),
$$

where $\lambda \in[0,1)$. Then, the following hold:

(i) There exists a point $\xi_{\star} \in P$ such that $d\left(\xi_{\star}, f \xi_{\star}\right)=d(P, Q)$;

(ii) $\xi_{\star}$ is a fixed point of $f^{2}$ in $P$ and $f \xi_{\star}$ is a fixed point of $f^{2}$ in $Q$;

(iii) For any $\xi_{0} \in P$, let $\xi_{n+1}=f \xi_{n}$. Then, the sequence $\left(\xi_{2 n}\right)$ converges to $\xi_{\star}$, and the sequence $\left(\xi_{2 n+1}\right)$ converges to $f \xi_{\star}$.

In the following, we give a result from Corollary 1 for nonexpansive mappings in normed vector spaces.

Theorem 7. Let $X$ be a normed vector space and $P, Q$ be two nonempty subsets of $X$. Given $f: P \rightarrow Q$ and $g: Q \rightarrow P$ are non-self mappings such that for all $(x, y) \in P_{0} \times Q_{0}$,

$$
\|f x-g y\| \leq\|x-y\|,
$$

where $P_{0}$ and $Q_{0}$ are defined by (1) and (2), respectively. Suppose that:

(i) $\varnothing \neq P_{0}$ is convex and boundedly compact;

(ii) $Q_{0}$ is compact; 
(iii) The functions $z \rightarrow\|z-f z\|$ and $z \rightarrow\|z-g z\|$ are lower semi-continuous in $P_{0}$ and $Q_{0}$, respectively. Then, there exists $\left(\xi_{\star}, \vartheta_{\star}\right) \in P_{0} \times Q_{0}$ such that:

$$
\left\|\xi_{\star}-f \xi_{\star}\right\|=\left\|\vartheta_{\star}-g \vartheta_{\star}\right\|=d(P, Q) .
$$

Proof. Since $P_{0} \neq \varnothing$, there exists $\left(\xi_{0}, \vartheta_{0}\right) \in P_{0} \times Q_{0}$ such that $\left\|\xi_{0}-\vartheta_{0}\right\|=d(P, Q)$. We claim that $f: P_{0} \rightarrow Q_{0}$ and $g: Q_{0} \rightarrow P_{0}$. Let $x \in P_{0}$, so there exists $y \in Q_{0}$, such that $\|x-y\|=d(P, Q)$. From (13),

$$
d(P, Q) \leq\|f x-g y\| \leq\|x-y\|=d(P, Q),
$$

which implies that $\|f x-g y\|=d(P, Q)$, and so, $f\left(P_{0}\right) \subset Q_{0}$. Similarly, we show that $g\left(Q_{0}\right) \subset P_{0}$.

For $n \geq 1$, consider:

$$
\begin{cases}f_{n} x=\frac{1}{n} \vartheta_{0}+\left(1-\frac{1}{n}\right) f x & \text { for } x \in P_{0}, \\ g_{n} y=\frac{1}{n} \xi_{0}+\left(1-\frac{1}{n}\right) g y & \text { for } y \in Q_{0} .\end{cases}
$$

Since $P_{0}$ is convex, we have that $g_{n}: Q_{0} \rightarrow P_{0}$. Again, for $x \in P_{0 \prime}$, there exists $y \in Q_{0}$ such that $\|x-y\|=d(P, Q)$. From (13),

$$
\begin{aligned}
d(P, Q) \leq\left\|f_{n} x-g_{n} y\right\| & \leq \frac{1}{n}\left\|\xi_{0}-\vartheta_{0}\right\|+\left(1-\frac{1}{n}\right)\|f x-g y\| \\
& \leq \frac{1}{n}\left\|\xi_{0}-\vartheta_{0}\right\|+\left(1-\frac{1}{n}\right)\|x-y\|=d(P, Q),
\end{aligned}
$$

which implies that $\left\|f_{n} x-g_{n} y\right\|=d(P, Q)$, and so, $f_{n} x \in Q_{0}$, that is $f_{n}: P_{0} \rightarrow Q_{0}$.

Let $(x, y) \in P_{0} \times Q_{0}$. Then:

$$
\begin{aligned}
\left\|f_{n} x-g_{n} y\right\| & \leq \frac{1}{n}\left\|\xi_{0}-\vartheta_{0}\right\|+\left(1-\frac{1}{n}\right)\|f x-g y\| \\
& \leq\left(1-\frac{1}{n}\right)\|x-y\|+\frac{1}{n} d(P, Q) .
\end{aligned}
$$

Since $\left(P_{0}, Q_{0}\right)$ is proximally complete, by Corollary 1 , there exists $\left(\xi_{n}, \vartheta_{n}\right) \in P_{0} \times Q_{0}$ such that:

$$
\left\|\xi_{n}-f_{n} \xi_{n}\right\|=\left\|\vartheta_{n}-g_{n} \vartheta_{n}\right\|=\left\|\xi_{n}-\vartheta_{n}\right\|=d(P, Q) \quad \text { for } n \in \mathbb{N}^{*} .
$$

We have:

$$
\left\|\xi_{n}-f \xi_{n}\right\| \leq\left\|\xi_{n}-f_{n} \xi_{n}\right\|+\frac{1}{n}\left\|\vartheta_{0}-f \xi_{n}\right\|
$$

Since $f \xi_{n} \in Q_{0}$ and $Q_{0}$ is compact, we get:

$$
\lim _{n \rightarrow \in \infty}\left\|\xi_{n}-f \xi_{n}\right\|=d(P, Q)
$$

Again,

$$
\left\|\xi_{n}\right\| \leq\left\|\xi_{n}-f_{n} \xi_{n}\right\|+\frac{1}{n}\left\|\vartheta_{0}\right\|+\left(1-\frac{1}{n}\right)\left\|f \xi_{n}\right\|=d(P, Q)+\left\|\vartheta_{0}\right\|+\left\|f \xi_{n}\right\|,
$$

which implies that $\left(\xi_{n}\right)$ is bounded. Since $P_{0}$ is boundedly compact, there exist $\xi_{\star} \in P_{0}$ and $\left(\xi_{n_{k}}\right)$ a subsequence of $\left(\xi_{n}\right)$ such that $\lim _{k \rightarrow \infty} \xi_{n_{k}}=\xi_{\star}$. From (14) and Assumption (iii), we have:

$$
\left\|\xi_{\star}-f \xi_{\star}\right\| \leq \liminf _{k \rightarrow \infty}\left\|\xi_{n_{k}}-f \xi_{n_{k}}\right\|=\lim _{k \rightarrow \infty}\left\|\xi_{n_{k}}-f \xi_{n_{k}}\right\|=d(P, Q),
$$

which implies that $\left\|\xi_{\star}-f \xi_{\star}\right\|=d(P, Q)$. 
On the other hand, we have:

$$
\begin{aligned}
\left\|\vartheta_{n}-g \vartheta_{n}\right\| & \leq\left\|\vartheta_{n}-g_{n} \vartheta_{n}\right\|+\frac{1}{n}\left(\left\|\xi_{0}\right\|+\left\|g \vartheta_{n}\right\|\right) \\
& \leq d(P, Q)+\frac{1}{n}\left(\left\|\xi_{0}\right\|+\left\|f \xi_{n}-g \vartheta_{n}\right\|+\left\|f \xi_{n}\right\|\right) \\
& \leq d(P, Q)+\frac{1}{n}\left(\left\|\xi_{0}\right\|+\left\|\xi_{n}-\vartheta_{n}\right\|+\left\|f \xi_{n}\right\|\right) \\
& =d(P, Q)+\frac{1}{n}\left(\left\|\xi_{0}\right\|+d(P, Q)+\left\|f \xi_{n}\right\|\right) .
\end{aligned}
$$

This implies that:

$$
\lim _{n \rightarrow \infty}\left\|\vartheta_{n}-g \vartheta_{n}\right\|=d(P, Q) .
$$

Notice that $\left(\vartheta_{n}\right)$ is bounded because $\left\|\xi_{n}-\vartheta_{n}\right\|=d(P, Q)$ and $\left(\xi_{n}\right)$ is bounded. Since $Q_{0}$ is compact, there exist $\vartheta_{\star} \in Q_{0}$ and a subsequence $\left(\vartheta_{n_{k}}\right)$ of $\left(\vartheta_{n}\right)$ such that $\lim _{k \rightarrow \infty} \vartheta_{n_{k}}=\vartheta_{\star}$. By assumption (iii), we have $\left\|\vartheta_{\star}-g \vartheta_{\star}\right\|=d(P, Q)$.

As particular cases from Theorem 7, we have:

Corollary 7. Let $X$ be a normed vector space and $P, Q$ be two nonempty subsets of $X$. Let $f: P \cup Q \rightarrow P \cup Q$ be a non-self map such that $f(P) \subseteq Q, f(Q) \subseteq P$ and for all $(x, y) \in P_{0} \times Q_{0}$,

$$
\|f x-f y\| \leq\|x-y\| .
$$

Suppose that:

(i) $\varnothing \neq P_{0}$ is convex and boundedly compact;

(ii) $Q_{0}$ is compact;

(iii) The function $z \rightarrow\|z-f z\|$ is lower semi-continuous in $P_{0}$.

Then, there exists $\xi_{\star} \in P_{0}$ such that:

$$
\left\|\xi_{\star}-f \xi_{\star}\right\|=d(P, Q) .
$$

Corollary 8. Let $X$ be a normed vector space and $P, Q$ be two nonempty subsets of $X$. Let $f: P \cup Q \rightarrow P \cup Q$ be a non-self map such that $f(P) \subseteq Q, f(Q) \subseteq P$ and for all $(x, y) \in P_{0} \times Q_{0}$,

$$
\|f x-f y\| \leq\|x-y\| .
$$

Suppose that:

(i) $\varnothing \neq P_{0}$ is convex and boundedly compact;

(ii) $Q_{0}$ is compact;

(iii) The function $z \rightarrow\|z-f z\|$ is lower semi-continuous in $Q_{0}$.

Then, there is $\vartheta_{\star} \in Q_{0}$ such that:

$$
\left\|\vartheta_{\star}-f \vartheta_{\star}\right\|=d(P, Q) .
$$

Remark 1. Corollaries $2,3,5,6,7$, and 8 remain true by replacing $f: P \cup Q \rightarrow P \cup B$ with $f: A \times Q \rightarrow$ $P \times B$ (keeping other hypotheses). 


\section{Conclusions}

In this paper, we considered proximally-complete pairs and proximally-complete semi-sharp proximinal pairs as weaker hypotheses with respect to [3] to get convergence and best proximity points. We applied Theorem 5 to provide a result for nonexpansive mappings in normed vector spaces.

Author Contributions: All authors contributed equally in writing this article. All authors read and approved the manuscript.

Funding: There was no external funding for this work.

Acknowledgments: This work has been financially supported by the Deanship of Scientific Research in King Faisal University, Project Number (180080). The authors are gratefully for this support. The authors also thank the reviewers for careful reading of the paper and for helpful comments, allowing us to improve it.

Conflicts of Interest: The authors declare no conflict of interest.

\section{References}

1. Aydi, H.; Felhi, A. On best proximity points for various $\alpha$-proximal contractions on metric-like spaces. J. Nonlinear Sci. Appl. 2016, 9, 5202-5218. [CrossRef]

2. Aydi, H.; Felhi, A. Best proximity points for cyclic Kannan-Chatterjea-Ćirić type contractions on metric-like spaces. J. Nonlinear Sci. Appl. 2016, 9, 2458-2466. [CrossRef]

3. Basha, S. Best proximity points: global optimal approximate solutions. J. Glob. Optim. 2011, 49, $15-21$. [CrossRef]

4. Basha, S.; Veeramani, P. Best approximations and best proximity pairs. Acta Sci. Math. 1997, 63, $289-300$.

5. Basha, S.; Veeramani, P. Best proximity pair theorems for multifunctions with open fibres. J. Approx. Theory 2000, 103, 119-129. [CrossRef]

6. Basha, S. Best proximity point theorems. J. Approx. Theory 2011, 163, 1772-1781. [CrossRef]

7. Choudhury, B.S.; Metiya, N.; Postolache, M.; Konar, P. A discussion on best proximity point and coupled best proximity point in partially ordered metric spaces. Fixed Point Theory Appl. 2015, 2015, 170. [CrossRef]

8. Di Bari, C.; Suzuki, T.; Vetro, C. Best proximity points for cyclic Meir-Keeler contractions. Nonlinear Anal. 2008, 69, 3790-3794. [CrossRef]

9. Espínola, R.; Kosuru, G.G.R.; Veeramani, P. Pythagorean property and best proximity point theorems. J. Optim. Theory Appl. 2015, 164, 534-550. [CrossRef]

10. Haddadi, M.R. Best proximity point iteration for nonexpensive mapping in banach spaces. J. Nonlinear Sci. Appl. 2014, 7, 126-130. [CrossRef]

11. Jacob, G.K.; Postolache, M.; Marudai, M.; Raja, V. Norm convergence iterations for best proximity points of non-self non-expansive mappings. UPB Sci. Bull. Ser. A 2017, 79, 49-56.

12. Jleli, M.; Karapinar, E.; Samet, B. Best proximity points for generalized $\alpha-\psi$-proximal contractive type mappings. J. Appl. Math. 2013, 2013, 534127. [CrossRef]

13. Karpagam, S.; Agrawal, S. Best proximity points theorems for cyclic Meir-Keeler contraction maps. Nonlinear Anal. 2011, 74, 1040-1046. [CrossRef]

14. Karpagam, S.; Agrawal, S. Existence of best proximity points of p-cyclic contractions. Fixed Point Theory 2012, 13, 99-105.

15. Kim, W.K.; Kum, S.; Lee, K.H. On general best proximity pairs and equilibrium pairs in free abstract economies. Nonlinear Anal. 2008, 68, 2216-2227. [CrossRef]

16. Aydi, H.; Felhi, A.; Karapinar, E. On common best proximity points for generalized $\alpha-\psi$-proximal contractions. J. Nonlinear Sci. Appl. 2016, 9, 2658-2670. [CrossRef]

17. Aydi, H.; Karapinar, E.; Salimi, P.; Erhan, I.M. Best proximity points of generalized almost $\psi$-Geraghty contractive non-self mappings. Fixed Point Theory Appl. 2014, 2014, 32. [CrossRef]

18. Kirk, W.A.; Reich, S.; Veeramani, P. Proximinal retracts and best proximity pair theorems. Numer. Funct. Anal. Optim. 2003, 24, 851-862. [CrossRef]

19. Kosuru, G.S.R.; Veeramani, P. On existence of best proximity pair theorems for relatively nonexpansive mappings. J. Nonlinear Convex Anal. 2010, 11, 71-77.

20. Kumam, P.; Aydi, H.; Karapinar, E.; Sintunavarat, W. Best proximity points and extension of Mizoguchi-Takahashi's fixed point theorems. Fixed Point Theory Appl. 2013, 2013, 242. [CrossRef] 
21. Mongkolkeha, C.; Kumam, P. Best proximity point Theorems for generalized cyclic contractions in ordered metric Spaces. J. Optim. Theory Appl. 2012, 155, 215-226. [CrossRef]

22. Nashine, H.K.; Kumam, P.; Vetro, C. Best proximity point theorems for rational proximal contractions. Fixed Point Theory Appl. 2013, 2013, 95. [CrossRef]

23. Omidvari, M.; Vaezpour, S.M.; Saadati, R. Best proximity point theorems for F-contractive non-self mappings. Miskolc Math. Notes 2014, 15, 615-623. [CrossRef]

24. Raj, V.S. Best proximity point theorems for non-self mappings. Fixed Point Theory 2013, 14, 447-454.

25. Raj, V.S.; Veeramani, P. A best proximity theorems for weakly contractive non-self mappings. Nonlinear Anal. 2011, 74, 4804-4808.

26. Prolla, J.B. Fixed point theorems for set-valued mappings and existence of best approximants. Numer. Funct. Anal. Optim. 1983, 5, 449-455. [CrossRef]

27. Sehgal, V.M.; Singh, S.P. A generalization to multifunctions of Fan's best approximation theorem. Proc. Am. Math. Soc. 1988, 102, 534-537.

28. Sehgal, V.M.; Singh, S.P. A theorem on best approximations. Numer. Funct. Anal. Optim. 1989, 10, $181-184$. [CrossRef]

29. Shatanawi, W. Best proximity point on nonlinear contractive condition. J. Phys. Conf. Ser. 2013, 435, 012006. [CrossRef]

30. Shatanawi, W.; Pitea, A. Best Proximity Point and Best Proximity Coupled Point in a Complete Metric Space with (P)-Property. Filomat 2015, 29, 63-74. [CrossRef]

31. Souyah, N.; Aydi, H.; Abdeljawad, T.; Mlaiki, N. Best proximity point theorems on rectangular metric spaces endowed with a graph. Axioms 2019, 8, 17. [CrossRef]

32. Vetrivel, V.; Veeramani, P.; Bhattacharyya, P. Some extensions of Fan's best approximation theorem. Numer. Funct. Anal. Optim. 1992, 13, 397-402. [CrossRef]

33. Zhang, J.; Su, Y.; Cheng, Q. A note on "A best proximity point theorem for Geraghty-contractions". Fixed Point Theory Appl. 2013, 2013, 99. [CrossRef]

34. Berinde, V. Contracaii Generalizatii Aplicaii; Editura Cub Press: Baia Mare, Romania, 1997; Volume 22.

(C) 2019 by the authors. Licensee MDPI, Basel, Switzerland. This article is an open access article distributed under the terms and conditions of the Creative Commons Attribution (CC BY) license (http:/ / creativecommons.org/licenses/by/4.0/). 